\title{
A Case Study of Pinduoduo Strategy Based on SWOT Analysis
}

\author{
Minyi $\mathrm{Li}^{1, *}$ Chengchung Tsai ${ }^{2}$ Lobel Trong Thuy $\operatorname{Tran}^{1}$ \\ ${ }^{1}$ Faculty of Business Administration, Ton Duc Thang University, Ho Chi Minh 800010, Vietnam \\ ${ }^{2}$ School of Management, Putian College, Putian, Fujian 351100, China \\ *Corresponding author. Email: 201901002@student.tdtu.edu.vn
}

\begin{abstract}
In less than five years, Pinduoduo, listed on National Association of Securities Dealers Automated Quotations (NASDAQ) in the United States, created a remarkable business legend. Within just two years, corporate Gross Merchandise Volume (GMV) broke through RMB 100 billion and Pinduoduo announced its profit for the first time in the financial report of the third quarter of 2020, and these dazzling performance have to make people go deep into its governance strategy to deal with market changes and the marketing strategy behind Pinduoduo. With that in mind, this paper mainly focuses on Pinduoduo's actions, organizational structure, succession plan, and implemented strategies, analyzes its external market and macro-environment and reviews its performance, and finally summarizes Pinduoduo's external and internal advantages and disadvantages by SWOT analysis, so as to put forward the corresponding strategy choice.
\end{abstract}

Keywords: Pinduoduo, Social e-commerce, Strategy, SWOT.

\section{INTRODUCTION}

Pinduoduo, founded by Huang Zheng in April 2015, is a third-party Chinese social e-commerce platform focusing on the Customer-toManufacturer (C2M), and an innovative ecommerce platform combining group-buying and social media. Pinduoduo has always been committed to creating the value of "cost-effective good stuff" for consumers. The company mainly sells affordable daily necessities and agricultural products. Users share Pinduoduo's links to friends and family members on social platforms for group buying. The more people who join the group, the cheaper the product price. Pinduoduo was officially listed on the NASDAQ Stock Exchange under the ticker symbol PDD on July 26, 2018. By the end of 2020 , the total market value of Pinduoduo has reached $\$ 200$ billion. In just three years, Pinduoduo has broken the bipolar pattern of China's ecommerce industry, and has been favored by many investors due to its high growth rate.

\section{STRATEGY}

The strategies implemented by Pinduoduo are as follows.

\subsection{Implementing the Development Strategy of "Encircling the City from the Countryside"}

That is, Pinduoduo takes users in less developed areas of China as a breakthrough point, and then users have slowly developed from rural consumers in the early stage to middle- and low-end consumers in some first-tier big cities today. Within six years, Pinduoduo has successfully acquired 730 million annual active users with an average annual consumption of nearly $\$ 300$ per user.

\subsection{Low-cost Leading Strategy}

First, Pinduoduo reduces intermediate links and the cost of goods by infinitely being close to the manufacturers. Second, Pinduoduo mainly sells unbranded products. Pinduoduo encourages many unbranded but price-competitive and guaranteed products to be sold on the platform, as these 
products basically satisfy the usage function and are cost-effective. Finally, Pinduoduo reduces logistics costs by delivering bulk commodities. Since orders are shipped in large quantities, sellers have greater bargaining power for logistics, and express delivery costs can naturally be lowered, which increases consumer surplus.

\subsection{Competitive Strategy of Strategic Loss}

It refers to the actual sacrifices made to obtain future benefits through a large amount of early marketing cost investment and tens of billions of subsidies. The basis for strategic loss is that Pinduoduo attracts users' attention and expands market share through large subsidies and low prices. Prior corporate net profit was all negative, but after the economic scale was formed, the first profit was achieved in the third quarter of 2020.

\subsection{The Operation Strategy of Increasing the Number of Users and Repurchasing Rate}

The new users come partly from China's rural market and partly from billions of dollars in subsidies. For price sensitive users, Pinduoduo meets the needs of daily consumption, or even upgrading consumption, while for users with strong spending power, Pinduoduo meets the need of price comparison in pursuit of cost performance of daily consumer goods, and also the platform game entertainment design as well as the accurate merchandise launch is to stimulate the user's repeated purchase desire. Therefore, the customer unit price has never been the core driving force of Pinduoduo's revenue growth, but the repurchase of users.

Pinduoduo's profit model is the volume difference model. Pinduoduo's main revenue comes from online advertising and transaction commissions. Online marketing revenue includes merchants bidding for advertising booths and traffic entrances. Transaction commission refers to the $0.6 \%$ handling fee paid by merchants to third-party payment providers. Through brand advertising and huge subsidies to obtain user traffic, this is the marketing and sales costs, plus other costs and expenses constitute the total expenditure. The growth rate of Pinduoduo's expenses and revenues shows a scissors gap, and the company is in a profitable state when the revenue exceeds the expenses. Pinduoduo's profit model is consistent with the current business models of other ecommerce platforms. ("Figure 1")

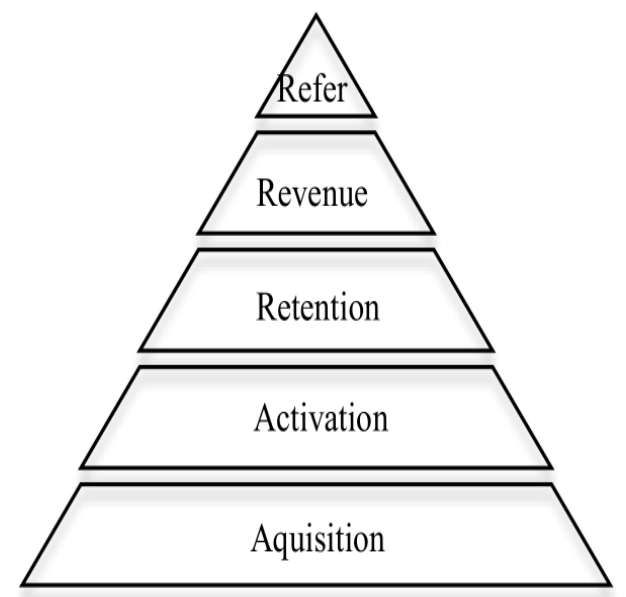

Figure 1 Pinduoduo AARRR user growth model.

\section{ACTIONS}

\subsection{To Invest a Huge Amount of Money for Advertising}

In 2018, Pinduoduo spent billions on advertising, sponsored many popular variety shows such as "Happy Camp" and "Magic City", and also sponsored CCTV's World Cup advertising. Although these huge investments have brought large short-term performance losses to Pinduoduo, from a long-term perspective, it has increased Pinduoduo's brand recognition.

\subsection{To Launch a Billion-dollar Subsidy Program}

After building a wide range of brand awareness through large-scale advertising and marketing, Pinduoduo teamed up with brands to launch a billion-dollar subsidy in 2019, and vigorously promoted the program at the most important promotion node to attract some pursuing brands cost-effective white-collar users. The billion-dollar subsidy program has not only attracted a large number of new users to Pinduoduo, but also improved consumers' psychological perception of Pinduoduo.

\subsection{To Create a "New Brand Plan"}

The core content of the plan is that Pinduoduo's platform provides data support, R\&D advice and traffic resources, and unites powerful factories and foreign trade companies to explore customized $\mathrm{C} 2 \mathrm{M}$ production based on consumer needs. On the 
one hand, it helps these companies gradually realize the transformation from brand manufacturers to owning their own brands, and on the other hand, it provides consumers with abundant, high-quality, low-priced and personalized products. For this reason, Pinduoduo can use these new brands to gain differentiated advantages and enhance competitiveness.

\subsection{To Design Lots of Interactive Games}

Buying by participating in the game both makes the user feel cheap and makes the bargain reasonable. Inviting friends to help is the core gameplay of Pinduoduo. When users buy goods, they forward the "bargain" link to their friends. The more friends who participate in bargaining, the lower the price of the product. In the gameplay of inviting friends to help, Pinduoduo has added shopping games such as "Duo Duo Orchard" and "Duo Duo Farm", which greatly stimulated users' interest and fun in shopping.

\section{CURRENT SITUATION AND TRENDS}

China's e-commerce transaction volume continues to expand. In recent years, the transaction scale of China's e-commerce industry has continued to expand, ranking first in the global online retail market. According to data from the National Bureau of Statistics of China, the value of ecommerce transactions in 2008 was only $\$ 0.48$ trillion, reached $\$ 1.5$ trillion in 2013 , and exceeded $\$ 4.6$ trillion in 2018, a year-on-year increase of $8.5 \%$. During the same period, China's online retail transaction volume has also grown rapidly at a rate of more than $40 \%$. In 2016, China's e-commerce has entered a relatively stable development period from a period of ultra-high-speed growth. In July 2020, the "China E-Commerce Development Report 2019-2020" pointed out that China's ecommerce transaction volume in 2019 was \$5.37 trillion, with an increase of $6.7 \%$ year-on-year.

\subsection{China's Rural E-commerce Is Well Developed}

During the same period, China's rural online retail has grown rapidly. In 2015, China's rural ecommerce transaction volume reached $\$ 0.054$ trillion, and in 2017 it exceeded \$1 trillion, an 18fold increase in transaction volume compared to 2015 's. In 2019, rural online retail sales reached $\$ 2.62$ trillion, a year-on-year increase of $19.1 \%$, which was $2.6 \%$ higher than the national online retail sales growth. E-commerce has achieved remarkable results in poverty alleviation. The ecommerce platform has helped China's 832 national poverty-stricken counties achieve poverty alleviation tasks.

\subsection{Large-scale Employment in China's E- commerce}

In recent years, the employment scale of ecommerce is growing, and the integration of ecommerce and real economy is accelerating, which leads more people to engage in e-commerce-related work. According to data from the China Internet Economic Research Institute, in 2018, there were 47 million e-commerce employees in China, a yearon-year increase of $10.6 \%$. The data for 2019 exceeded 50 million people.

\subsection{Continuous Growth of E-commerce Users}

With the popularization of smart phones and the rapid development of the mobile Internet, the scale of online shopping users in China continues to expand. In 2013, there were only 300 million online shopping users in China. In 2018, the number of online shopping users exceeded 600 million. In the first half of 2019, China's online shopping market maintained rapid development. The rural market and cross-border e-commerce provided new growth momentum for the online shopping market. In terms of regions, markets represented by small and medium-sized cities and rural areas have expanded the room for growth in online consumption. As of June 2019, the number of online shopping users in China reached 739 million, accounting for $74.8 \%$ of the total Internet users, an increase of $21 \%$ from the end of 2018.

It can be seen from the above data that China's e-commerce market has a good development prospect, and the corresponding employment, users and transaction volume continue to grow. Rural ecommerce has also become an emerging hot spot in recent years.

\section{COMPETITION}

The homogeneity competition in China's ecommerce industry is fierce. In the industry chain of China's mobile e-commerce platform, it can be divided into general e-commerce, vertical commodity e-commerce, cross-border e-commerce, 
shopping guide e-commerce, etc. In addition, the mobile e-commerce industry chain also includes mobile payment and e-finance, such as Alipay, WeChat Pay, JD Baitiao, etc. The logistics chain includes SF Express, JD Logistics, Cainiao Logistics, etc., and second-hand e-commerce platforms such as Xianyu, Paipai Second-hand, Zhuan Zhuan, etc. In general, China has formed a pattern in which multiple e-commerce models coexist, and the e-commerce industry chain is relatively complete. Pinduoduo relies on its unique advantages to occupy a certain position in China's e-commerce. However, due to the overlap of products and customer groups, it still faces challenges from many existing and potential competitors.

Pinduoduo's competitors: according to the data of China's e-commerce retail market share in 2019, Alibaba took the first place with $58.2 \%$, followed by JD.com with $17.3 \%$. These two e-commerce giants accounted for nearly $80 \%$ of China's ecommerce market share. The fast-growing Pinduoduo accounts for only $5.2 \%$, so there is still a large room for market share to rise. In addition, Pinduoduo's direct competitor is Alibaba. Alibaba has a relatively complete ecosystem and has developed four softwares including Taobao, Tmall, Taobao Special Edition, and Yitao with different positioning. Tmall focuses on high-quality products and Taobao focuses on mid-end products. Taobao Special Edition is an e-commerce platform that responds to Pinduoduo's low-price strategy. Yitao is a shopping navigation portal for coupons and rebates, which satisfies consumers of different levels to the greatest extent.

In general, the differentiated positioning strategy adopted by Pinduoduo has avoided many positive competitions, and the initial competitive environment is relatively friendly, which is also one of the external conditions for the rapid development of Pinduoduo. However, when the market in firstand second-tier cities is saturated in the future, Taobao, JD.com, and traditional e-commerce platforms will seek new growth points from underdeveloped regions. In the long run, Pinduoduo's competition within the industry will become fierce.

\section{MACRO-ENVIRONMENT}

\subsection{Legal and Policy Environment}

China's "E-commerce 15th Year Development Plan" proposes to actively create a relaxed environment for e-commerce entrepreneurship and promote the vitality of e-commerce. This policy drew a clear line for the e-commerce environment. With policy guidance, Pinduoduo's e-commerce platform can make more stable progress and development. Second, China has introduced supporting policies for rural e-commerce. For example, in 2018, the "Notice on Carrying out the Comprehensive Demonstration Work of Ecommerce in Rural Areas in 2018" was issued, which is closely related to the development of ecommerce in rural areas and is conducive to the realization of the connection between urban and rural production and consumption. This is also of great help to the poverty alleviation plan implemented by Pinduoduo in 2018, making full use of the social e-commerce model in operation to solve the problem of unsalable agricultural products through consumer orders.

\subsection{Economic Environment}

By 2020, China has fully achieved the goal of a well-off society, and China's domestic economy is expected to maintain a stable economic growth rate of 5\%. According to the National Bureau of Statistics of China, the growth rate of China's rural per capita consumption expenditure in 2019 was higher than that of urban residents' per capita consumption, which was $8.7 \%$. The increased disposable income has brought economic fundamental advantages to people's life, and the total retail sales in the underdeveloped areas in the entire market has been close to half. The potential huge consumption power of rural residents in China has become increasingly significant, which lays the foundation for Pinduoduo's low price strategy.

\subsection{Social Environment}

According to the 44th "Statistical Report on China's Internet Development Status", as of June 2019, China's Internet penetration rate has reached $61.2 \%$. Among them, the scale of online shopping users reached 739 million, the scale of mobile online shopping users reached 722 million, and the utilization rate of mobile online shopping software was as high as $73.4 \%$. It can be seen that Chinese mobile phone netizens account for a significant proportion of the overall number of netizens, and the scale of online shopping using mobile phones is also a significant proportion of the scale of online shopping, which shows that online shopping has become an essential daily activity for Chinese netizens. 


\subsection{Technological Environment}

In recent years, technologies such as big data, artificial intelligence, cloud computing, virtual reality technology, logistics chain and 4G network have developed rapidly, providing a good technical environment for e-commerce platforms such as Pinduoduo and effectively promoting the efficiency of e-commerce decision-making.

\section{TRENDS}

China's e-commerce market will enter a period of steady growth. Unlike the rapid growth period in the past six years, the development of China's ecommerce will slow down in the future, and the proportion of online shopping in social retail will steadily increase, there is still room for strategic entry.

\subsection{There Will Be a Merger Boom in China's E-commerce Platforms}

At present, China's online shopping market has a relatively high degree of industry concentration. The e-commerce platforms have the same or similar positioning and the same business content. Due to the expected limited growth of the e-commerce market in the future, the winners are mostly large and competitive e-commerce platforms. Therefore, these companies will adopt complementary acquisition strategies for small e-commerce companies with good development prospects and form strategic alliances. It will become an inevitable trend for different types of e-commerce companies to cooperate with each other in the form of strategic alliances.

\subsection{The Prosperity of $\mathrm{O} 2 \mathrm{O}$ and $\mathrm{C} 2 \mathrm{M}$ Models}

At present, Pinduoduo has made a good attempt in adopting the C2M model, and has gradually realized the transformation from industrial standard products to small batches of personalized customization. $\mathrm{O} 2 \mathrm{O}$ and $\mathrm{C} 2 \mathrm{M}$ rely on intelligent customized platforms. Rapidly innovating and iterating products based on the individual needs of users is the guarantee for the healthy and sustainable development of future e-commerce platforms.

\subsection{Rural E-commerce Will Usher in a Period of Rapid Development}

Favorable factors such as favorable policies, ecommerce platform replenishment, and rural mass Internet have promoted rural e-commerce to become an important growth point for the online market in the future. The penetration rate of online shopping in China's first- and second-tier cities is close to saturation, and e-commerce has gradually penetrated into third-, fourth- and fifth-tier cities. The layout of rural e-commerce will become the focus of future e-commerce enterprise development. Alibaba, JD.com, Pinduoduo and Meituan are all deploying rural businesses. In fact, the e-commerce platform that first seizes third- and fourth-tier cities will have a greater advantage in future competition.

\subsection{Mobile Social Shopping Will Become a New Trend}

In 2019, the scale of social retail users in China will reach 310 million, and the scale of social ecommerce transactions will reach 5.35 billion US dollars. The figure for the same period in 2018 was 3.38 billion US dollars, an increase of $58 \%$ yearon-year. Mobile social e-commerce is in line with the new trend of the future development of the Internet. Shopping has become a means of social interaction, meeting the needs of increasingly niche and personalized users through the sharing and interaction of social groups.

\section{PERFORMANCE REVIEW}

\subsection{Pinduoduo's Revenue}

On November 12, 2020, Pinduoduo released its financial report for the third quarter of 2020. The financial report showed that Pinduoduo's thirdquarter revenue was $\$ 2.19$ billion, an increase of $89.11 \%$ year-on-year. Under non-GAAP, Pinduoduo Duoduo's net profit was $\$ 72$ million, achieving quarterly profit for the first time. Based on past data, Pinduoduo is expected to maintain a growth rate of around $80 \%$ in 2021 . Among them, Pinduoduo's revenue has increased by 59 times from 2016 to 2019.

\subsection{Pinduoduo's Gross Merchandise Volume (GMV)}

In terms of GMV, Pinduoduo achieved a GMV breakthrough of $\$ 1.5$ billion in 2017. It took 10 
years for JD.com, 8 years for VIPshop, 5 years for Taobao, and 2 years for Pinduoduo. In 2019, Pinduoduo's GMV exceeded $\$ 15$ billion for the first time, reaching $\$ 15.54$ billion. However, the growth rate of GMV has slowed significantly in the past two years, with a year-on-year growth rate of $73 \%$ in the third quarter of 2020.

\subsection{Pinduoduo Annual Active Buyer Expenditure}

As of December 31, 2019, Pinduoduo's annual active buyer expenditure was $\$ 265$, a $52.6 \%$ increase from \$173 in the same period in 2018. While Alibaba's per capita GMV in the first quarter of 2019 was $\$ 1,352$, JD's per capita GMV in the fourth quarter of 2018 was \$847. Although Pinduoduo's annual active buyer expenditure continues to grow, there is still a large gap compared with the data of Alibaba and JD.

\subsection{Pinduoduo's Net Profit}

Most of Pinduoduo's operating expenses are spent on marketing. In the fourth quarter of 2019 , operating expenses reached $\$ 1.68$ billion, of which marketing expenses were $\$ 1.43$ billion. The marketing expense ratio has always remained at a high level and has declined in the past three quarters. Pinduoduo was in a state of loss until the third quarter of 2020, but starting in 2019, its net loss narrowed, and finally achieved profit for the first time in the third quarter of 2020, with net profit turning from negative to positive to $\$ 72$ million.

Overall, Pinduoduo's strategic loss strategy has achieved greater results. As the founder Huang Zheng said, "Pinduoduo has the ability to make money at any time, because the short-term expenses on the books are also extremely adjustable at any time." Considering the development history of several other e-commerce companies and Pinduoduo's own financing scale, Pinduoduo's conversion rate is the strongest among other ecommerce companies. Therefore, Pinduoduo's business model should be correspondingly affirmed based on past data and performance.

\section{THE ELEMENTS OF ITS MARKETING PROGRAM}

\subsection{Differentiated Marketing Strategy: Precise Target Consumer Groups}

When choosing the target market, Pinduoduo avoids the first- and second-tier cities chosen by competitors. Instead, it chooses small cities or towns with greater consumption potential. Pinduoduo uses differentiation to target users in third- and fourth-tier cities. The typical users of Pinduoduo are mostly low income, strong dependence on the network, and extremely price sensitive.

\subsection{Diversified Product Portfolio}

At the beginning of its founding, Pinduoduo chose low-priced and high-repurchase foods as its core products, and also planned a series of marketing activities such as "Bargain for Free" and "Duoduo Play", focusing on fresh food, daily necessities and other products with high repurchase rates.

\subsection{C2M Shopping Model of Group Buying}

Pinduoduo adopts the $\mathrm{C} 2 \mathrm{M}$ model, which is completely different from Alibaba's C2C and B2B. The $\mathrm{C} 2 \mathrm{M}$ model is a business activity determined and initiated by consumers. Pinduoduo chose the form of group buying as its shopping model, which is not only convenient for users and allows users to have a certain bargaining power, but also great for sellers, because concentrated orders enable merchants to reduce costs and inventory risks.

\subsection{Socialized E-commerce Model}

The socialized e-commerce model has brought sales traffic to Pinduoduo, and the fission-style sharing and promotion has helped Pinduoduo to gain popularity. Flash sale is a feature of Pinduoduo's promotion. Every time someone creates a shopping group, the interface will display a notification "XXX created a shopping group 1 second ago." Usually, the time for group joining will be counted down quickly, which effectively creates urgency. In this kind of shopping atmosphere, it increases the desire of buyers to buy quickly, and causes users to take advantage of the situation. 
To this end, Pinduoduo's marketing plan mainly focuses on the three key marketing dimensions of "low price stimulation, game design and social fission".

\section{EXTERNAL OPPORTUNITIES}

China has 600 million low-and middle-income groups, and the scale of price-sensitive consumers in lower-tier cities is huge. Most of them are very sensitive to commodity prices and love online shopping, forming a huge consumption scale. This is the market advantage that Pinduoduo has seized. At the beginning of its development, Pinduoduo entered the blue ocean market of low-income groups in China, at that time, competitors ignored the consumption contribution of low-income groups.

\subsection{A Large Number of Taobao Merchants Turned to Pinduoduo}

The upgrade of Alibaba and JD.com platforms forced low-end sellers to find new e-commerce platforms. Most of Pinduoduo's merchants are small and medium businesses that were abandoned by Taobao after Taobao's upgrade. Since 2015, Alibaba has gradually begun its anti-counterfeiting work. According to Alibaba's 2017 Annual Report on Alibaba's Intellectual Property Protection, 240,000 Taobao stores were closed for the entire year of 2017, and Pinduoduo has become the main platform to receive these online stores.

\subsection{The Chinese Government Highly Supports the Development of Rural E- commerce}

China vigorously supports the online sales of agricultural products from the policy level, and the development space for rural e-commerce will further increase. The development strategy advocated by Pinduoduo coincides with the national policy. The products displayed on the Pinduoduo's platform are mostly commodities with an average price of less than $\$ 5$, most of which are agricultural products and food. In recent years, Pinduoduo has made great contributions to poverty alleviation and assistance to farmers, and has become a strategic partner of China's national agricultural and poverty alleviation.

\section{EXTERNAL THREATS}

\subsection{External E-commerce Competitors Gradually Open up the Low-end Market}

After being aware of Pinduoduo's rapid development strategy, Chinese e-commerce giants such as Alibaba and JD.com respectively launched new low-price e-commerce platforms such as "Taobao Special Edition" and "Jingxi" in response to Pinduoduo's low-price threat. To a certain extent, Pinduoduo has thus lost some price-sensitive customers.

\subsection{The Rising Trend of Consumption Upgrading}

Pinduoduo's low-price marketing strategy runs counter to the trend of consumption upgrades. Although it can attract a large number of pricesensitive customer groups in the initial stage, with the increase in per capita income and consumption concepts, customer groups tend to pay more attention to quality rather than price than before. Therefore, Pinduoduo's products may face the possibility of being eliminated by the times.

\subsection{WeChat Restricts Inductive Sharing of Links}

In 2019, WeChat software released the "WeChat External Link Content Management Specification", which began to tighten the management of external links and restrict users from reposting inductive links. This has caused Pinduoduo, which relies on WeChat traffic, to encounter certain promotion resistance.

\section{INTERNAL STRENGTHS}

\subsection{Professional Management and Leadership}

The founder Huang Zheng has worked at Google in the U.S. and has 14 years of entrepreneurial experience. He has a very deep understanding of operations and user management, which makes Pinduoduo pays attention to the design choices of various operating strategies based on its business model. 


\subsection{Adopting Low-price Marketing Strategies}

Low-price marketing is one of Pinduoduo's core competitiveness, which has a huge appeal to most consumers. In addition, the low cost of opening stores for merchants provides consumers with many affordable products. It is precisely through lowpriced product marketing strategies that Pinduoduo has obtained a large source of revenue.

\subsection{Low Cost, High Conversion Rate Customer Growth Model}

Leveraging on WeChat's traffic base of 1 billion users, Pinduoduo has successfully accumulated early loyal users through a large number of shared links. Huge traffic exposure, low-cost advertising effects and high customer conversion rates have become Pinduoduo's great competitive advantages.

\subsection{Game Design to Stimulate Shopping Desire}

The importance of game interaction for user retention is reflected in the multiple interactive sections developed by Pinduoduo. On the one hand, these interactive sections effectively increase the user's usage time, and on the other hand, Pinduoduo further consolidate user retention.

\section{INTERNAL WEAKNESSES}

\subsection{The Variety of Goods Is Limited and the Quality Is Poor}

Pinduoduo has fewer commodity categories, and its low commodity prices bring poorer quality and product experience. Consumers are less satisfied with the quality of Pinduoduo's goods. Pinduoduo's rating on the Apple appstore is only 4, and negative reviews criticize Pinduoduo's lowquality and counterfeit products.

\subsection{High Marketing Costs}

Pinduoduo, which has grown up brutally with tens of billions of subsidies and WeChat traffic support, has achieved profitability in the third quarter of 2020, proving its once questioned profitability. But the slowdown in customer unit price and GMV growth has also released a sign that Pinduoduo's growth lacks momentum.

\subsection{Unbalanced User Structure}

Pinduoduo's user structure is too concentrated in low- and middle-income consumers in third- and fourth-tier cities, and the proportion of middle- and high-income consumers in first-tier cities is small. Correspondingly, its annual active buyer expenditure is less.

\subsection{Frequent Marketing Activities Disgust Consumers}

It is Pinduoduo's group buying activities that appear in front of the public too frequently, overconsuming users' interpersonal relationships and energy, so Pinduoduo's strategy of using social circles is also a double-edged sword.

\section{STRATEGIC CHOICE: SWOT}

\subsection{SO Strategy}

\subsubsection{The Management Should Focus on the Innovation of Business Model}

For example, for the rural market, it should do a good job in product portfolio planning, adopt a market strategy of "medium-low-price products as the mainstay, and high-end products as a supplement", and accumulate more popularity through low-price, multiple purchase marketing strategies.

\subsubsection{Strengthening the Investment and Construction of Rural E-commerce}

Pinduoduo should broaden the vertical supply chain of agricultural products, increase the construction of rural basic logistics, and improve capital investment in agriculture to expand Pinduoduo's layout in rural e-commerce.

\subsubsection{Developing the Commercial Value of Existing Users}

Pinduoduo should make full use of the huge user traffic to increase new profit points, such as launching financial microfinance, wealth management, purchasing services and other services to create revenue, and apply marketing strategies that can improve company performance. 


\subsection{ST Strategy}

\subsubsection{Establishing a New Retail Digital Model}

It can use advanced technology such as big data and artificial intelligence to upgrade the production, circulation and sales process of platform products, and sell high-quality products according to consumers' preferences. Besides, Pinduoduo should expand the establishment of offline experience stores to achieve online and offline integration in order to improve consumers' shopping experience.

\subsubsection{Paying Close Attention to the \\ Development of the E-commerce Industry and Competitors}

From the business point of view, Pinduoduo's competitive advantages and value are identified, and differentiated competitive strategies are implemented in accordance with market demand. Secondly, it is possible to establish a multi-party cooperation and win-win relationship to increase external high-quality resources, achieve complementary advantages between enterprises, and avoid vicious competition.

\subsubsection{Consolidating and Improving Customer Loyalty}

It needs to strengthen emotional exchanges with users based on user needs, such as holding free training activities, establishing a multiple consumptions reward system, providing users with gifts, discounts and other benefits, while focusing on the construction of platform customer service centers, timely and correct dealing with user problems properly is conducive to improving user satisfaction.

\subsection{WO Strategy}

\subsubsection{Focusing on Branding Strategy}

It's necessary to through the establishment and development of the platform to strengthen Pinduoduo's brand effect, and implement a comprehensive brand strategy to enhance customer brand awareness. For example, the brand pavilion tried by Pinduoduo gradually shifted traffic to brand merchants after upgrading the management ability of platform merchants.

\subsubsection{Improving Product Quality \\ Supervision System}

Pinduoduo should conduct stricter source supervision on all categories of goods on the platform, such as regularly checking whether the quality of products supplied by merchants is qualified and blacklisting merchants that sell counterfeit products to combat counterfeit sales. In terms of quality management, it is necessary to transparently strengthen the random inspection and evaluation of the quality of enterprises.

\subsubsection{Introducing Well-known Brands That Are Popular with High-income People}

Pinduoduo can sign long term strategic agreements with these well-known brands and give them enough exposure to attract high-income consumers to spend on Pinduoduo.

\subsection{WT Strategy}

\subsubsection{Continuing to Develop the Existing C2M Model}

It can cooperate with brand and non-brand manufacturers, continuously upgrade the product supply chain, and do accurate identification and recommendation to provide consumers with more diversified products.

\subsubsection{Independently Establishing Low-cost Promotion Channels}

Pinduoduo can establish accounts on wellknown social platforms such as Weibo, Zhihu, and Maimai, and promote its marketing activities through widespread social media communication. Secondly, relevant staff must strictly control the marketing process and costs, and reasonably control marketing expenses.

\subsubsection{Gradually Changing Marketing Methods}

Pinduoduo can set up special discount festivals from the perspective of improving user experience, such as setting up discount festivals for fresh fruits and agricultural products or rebating consumers through daily check-in, so as to enhance consumers' trust in Pinduoduo. 


\section{CONCLUSION}

From an operational perspective, Pinduoduo's strategy is successful. Strategic aggressiveness and tactical pragmatism are the root causes of Pinduoduo's achievements in just a few years. As a dark horse in China's e-commerce, Pinduoduo has applied its unique business strategy to activate the market potential of the new social e-commerce and occupy a place in the Chinese e-commerce industry.

The rise of Pinduoduo is essentially the transformation of shopping experience from intent to scene. The increase in the duration of the mobile Internet actually marks a decrease in our initiative. The large-scale popularization of AI-based accurate algorithm push has reduced the ability of consumers to actively choose online. Only $42 \%$ of Pinduoduo users use the search function, and most of the users' consumption needs are motivated by sharing links, page pushes and discounts. Nowadays, users have mostly already got rid of temporary shopping intentions, but converted into consumption when a certain scene is recommended or pushed by the platform.

But Pinduoduo also has many problems to be solved. There are still shortcomings in terms of commodities and supervision, and Pinduoduo are facing the dilemma of low customer unit prices and high marketing expenses. Problems such as many fakes and poor commodities also have seriously affected the reputation of Pinduoduo. Secondly, the external environment has become more and more severe, Tencent's promotion traffic is restricted, and e-commerce giants such as Taobao and JD.com are paying more and more attention to the low- and middle-income market. How to better respond to the increasingly fierce competition in e-commerce and rapidly changing market demand is a question that Pinduoduo need to focus on in the next phase.

Pinduoduo still has long-term development potential. The senior management of Pinduoduo is currently focusing on formulating the company's long-term strategy. It plans to stabilize and strengthen Pinduoduo's development advantages from the adjustment of the corporate organizational structure and governance. But at the same time, Pinduoduo is clearly taking the path of first development and then rectification. Therefore, continuing to find new profit growth points and be committed to establishing a sustainable business model is the first priority for Pinduoduo in the future.

\section{AUTHORS' CONTRIBUTIONS}

Minyi $\mathrm{Li}$ is responsible for data analysis and paper writing, Chengchung Tsai collected data and Lobel Trong Thuy Tran contributed to editing.

\section{REFERENCES}

[1] Porter, M. E. (1980). Competitive strategy: techniques for analyzing industries and competitors. Social Science Electronic Publishing(2), 86-87.

[2] Judy Young, Gail Ridley. (2017). E commerce in Developing Countries. The Electronic Journal of Information Systems in Developing Countries 11(1), 1-6.

[3] Muhammad Anwar, Syed Z. A. Shah. (2020). Entrepreneurial orientation and generic competitive strategies for emerging SMEs: Financial and nonfinancial performance perspective, Journal of Public Affairs, 18-25.

[4] Li Minyi. (2019). A study on corporate social responsibility in Macau's gambling Industry. 2019 Southeast Asian Business Management Seminar in Taiwan, 33-39.

[5] Li Minyi, Tsai Cheng-chung. (2019). Analysis on the transformation and management of Taiwan's universities with fewer children and difficult enrollment. 2019 2nd Thailand International Chinese Symposium, 43-49.

[6] Li Minyi, Liu Xiaojing, Tsai Cheng-chung. (2017). Viewing the trend of China's medical reform from the perspective of grassroots governance - The case of municipal conflicts in Sanming, Fujian province. 2017 4th Guangdong Social Work Conference, 22-28. 